\title{
Left Atrial Volume Is an Independent Predictor of All-Cause Mortality in Chronic Hemodialysis Patients
}

\author{
Junichi Shizuku ${ }^{1,2}$, Tetsuri Yamashita ${ }^{1,2}$, Takashi Ohba ${ }^{2}$, Takashi Kabaya ${ }^{2}$ and Kosaku Nitta ${ }^{1}$
}

\begin{abstract}
Objective An enlarged left atrium (LA) has recently been identified as a risk factor for adverse cardiovascular outcomes in various pathologic conditions. However, few studies have evaluated its prognostic value in hemodialysis (HD) patients.

Methods We conducted an observational study to investigate whether an enlarged LA predicted all-cause mortality in $174 \mathrm{HD}$ patients. Patients were stratified into two groups based on the LA volume index (LAVI) value of $32 \mathrm{~mL} / \mathrm{m}^{2}$.

Results An increased left atrial volume index (LAVI $\left.>32 \mathrm{~mL} / \mathrm{m}^{2}\right)$ was present in $28(16.1 \%)$ of the HD patients. During the follow-up period (50.1 \pm 22.4 months), 77 patients $(44.3 \%)$ died. A Kaplan-Meier analysis revealed that the 7-year survival rate was significantly lower in the group whose LAVI was $>32 \mathrm{~mL} / \mathrm{m}^{2}$ than in the group whose LAVI was $\leq 32 \mathrm{~mL} / \mathrm{m}^{2}$ ( $\mathrm{p}=0.0033$ ). Multivariate analyses adjusted for echocardiographic parameters and clinical and laboratory data showed that increased LAVI was an independent predictor of allcause mortality (hazard ratio 1.030, 95\% confidence interval 1.004-1.056, $\mathrm{p}=0.0260$ ). Moreover, increased LAVI had a higher predictive value for all-cause mortality (area under the receiver operating characteristic curve $=0.612, \mathrm{p}=0.0059$ ) among the measured echocardiographic parameters.

Conclusion The results of the present study suggested that measurement of LAVI may be helpful in the risk stratification of HD patients and in providing therapeutic direction for their management.
\end{abstract}

Key words: hemodialysis, echocardiography, left atrial volume, mortality

(Intern Med 51: 1479-1485, 2012)

(DOI: 10.2169/internalmedicine.51.7284)

\section{Introduction}

Cardiovascular disease (CVD) is a major complication in end-stage renal disease (ESRD) patients and is the cause of death in half of such patients $(1,2)$. Echocardiography is an established method for estimating the risk of CV complications and for guiding the treatment of hemodialysis (HD) patients (3). Three of the many parameters measured by this method, left ventricular mass index (LVMI), ejection fraction (EF), and LV chamber volume, are particularly helpful in the initial risk stratification of dialysis patients and in risk monitoring during the follow-up period (4).

An enlarged left atrium (LA) has recently been identified as another valuable parameter for predicting adverse $\mathrm{CV}$ outcomes. In general, the LA performs multiple functions, acting as a reservoir during LV systole, a conduit for blood passing from the pulmonary veins to the LV during early diastole, an active contractile chamber that augments LV filling in late diastole, and a suction pump that refills itself in early systole (5). The LA enlarges in response to two conditions: pressure overload and volume overload. The LA enlargement that occurs due to pressure overload is usually secondary to increased LA afterload in mitral valve disease or LV dysfunction. In the general population, an enlarged LA has been reported to predict adverse $\mathrm{CV}$ outcomes, such as atrial fibrillation, stroke, congestive heart failure, and CV death (6).

However, few studies have evaluated the prognostic value of an enlarged LA in hemodialysis (HD) patients. We conducted this observational cohort study to investigate whether an enlarged LA predicts an adverse outcome and to compare

${ }^{1}$ Department of Medicine, Kidney Center, Tokyo Women's Medical University, Japan and ${ }^{2}$ Dialysis Unit, Minami Senju Hospital, Japan Received for publication January 10, 2012; Accepted for publication February 29, 2012

Correspondence to Dr. Kosaku Nitta, knitta@kc.twmu.ac.jp 
its usefulness as a predictor with other echocardiographic parameters in the same cohort.

\section{Materials and Methods}

\section{Study population}

This study was conducted as a prospective, single-center cohort study over a 7-year follow-up period. The exclusion criteria were: malignancy, active infection, nonsinus rhythm, pericardial effusion, and evidence of major valvular heart disease. All subjects had been treated at the Minami Senju Hospital for more than 6 months and were receiving standardized HD prescriptions $(500 \mathrm{~mL} / \mathrm{min}$ dialysate flow; 200 $\mathrm{mL} / \mathrm{min}$ blood flow; 4 hours of dialysis per session; 3 sessions per week). The Institutional Research Ethics Committee approved the study protocol, and the study was conducted in compliance with the Declaration of Helsinki.

Of the $236 \mathrm{HD}$ patients, 174 (age: $63.8 \pm 12.1$ years, dialysis vintage: $8.7 \pm 8.6$ years, male/female $=113 / 61$ ) gave informed consent to participation in the study and underwent echocardiography between January 2005 and December 2008. The underlying renal disease was diabetic nephropathy in 71 patients $(48.6 \%)$, chronic glomerulonephritis in 65 patients, nephrosclerosis in 9 patients, polycystic kidney disease in 6 patients, and lupus nephritis in 3 patients and it was unknown in 20 patients. The demographic and clinical data, including age, gender, systolic and diastolic blood pressure (BP) were recorded. BP was measured three times after the subject had rested in the supine position for at least 10 minutes, and the average of the three measurements was adopted at the time of echocardiographic measurements. The definition of hypertension used was systolic BP >140 $\mathrm{mmHg}$ and/or diastolic BP $>90 \mathrm{mmHg}$ or taking antihypertensive agents. Body surface area (BSA) was calculated according to a simplified formula: $0.007184 \times$ weight ${ }^{0.425} \times$ height $^{0.725}$. CVD was defined as having a history of myocardial infarction, angina pectoris, congestive heart failure $(\mathrm{CHF})$, stroke or peripheral artery disease.

\section{Laboratory tests}

Blood was drawn in a fasting state just before the start of each dialysis session. Blood samples were collected after the patients had fasted for at least 9 hours. The serum levels of urea nitrogen, albumin, calcium, phosphate, glucose, and Creactive protein (CRP) and the hemoglobin concentration were measured by routine laboratory methods. The mean values of 3 measurements made during each of the 3 months before echocardiography were used in the analysis. Diabetes mellitus was diagnosed on the basis of World Health Organization criteria (7). Serum intact parathyroid hormone (iPTH) was measured once when echocardiography was performed. The $\mathrm{Kt} / \mathrm{V}$ ratio was used as a measure of clearance in which $\mathrm{K}$ is the urea clearance of the dialyzer, $\mathrm{t}$ is the duration of dialysis, and $\mathrm{V}$ is the volume of distribution of urea in the patient (8). Information on treatment with vitamin D3, angiotensin-converting enzyme inhibitors (ACEIs), angiotensin receptor blockers (ARBs), and lipidlowering agents was collected from the medical records.

\section{Echocardiography}

An experienced senior cardiosonographer performed the echocardiographic studies with an Aplio 50 (Toshiba, Tokyo, Japan) ultrasound imager equipped with a 2.2/4.4 MHz (harmonics) phased-array $3 \mathrm{~S}$ transducer during continuous electrocardiographic recording. The data were saved in the archive (digital archive, raw data), and the images obtained were recalled for calculation of the study parameters by the same interpreter, who was unaware of the patients' clinical status. To minimize the effect of fluid overload, the echocardiographic evaluation was performed after a dialysis session. The two-dimensional guided M-mode echocardiographic study of the LV was performed in the parasternal long-axis view, and LV end-systolic diameter (LVDs), LV enddiastolic diameter (LVDd), interventricular septum thickness (IVST), and posterior wall thickness (PWT) were recorded as the mean values measured in five consecutive cardiac cycles, in accordance with the recommendations of the American Society of Echocardiography (9). LV systolic function was determined based on the EF by using a modified biplane Simpson's method from apical two- and four-chamber views (10), and we divided the patients into two groups by $55 \%$ as a median value. LV mass was calculated by the method described by Devereux et al. (11). LVMI was calculated by dividing LV mass by BSA, and LV hypertrophy (LVH) was defined as an LVMI $>131 \mathrm{~g} / \mathrm{m}^{2}$ for men and $>$ $100 \mathrm{~g} / \mathrm{m}^{2}$ for women (12). LA diameter was measured in the parasternal long-axis view by making a leading edge-toleading edge measurement of the maximal distance between the end-systolic posterior aortic root wall and posterior LA wall in accordance with the American Society of Echocardiography guidelines (13). LA volume was assessed by the biplane area-length method from apical four-chamber and two-chamber views (14). Measurements were obtained in end systole from the frame preceding mitral valve opening, and the LA volume was indexed for BSA. According to the results of population-based studies, $32 \mathrm{~mL} / \mathrm{m}^{2}$ was considered the upper limit of the normal range of LA volume index (LAVI) values (15). Tissue Doppler mode was used to record mitral annulus velocities at the septal and lateral corners. Early diastolic (E), and late diastolic (A) tissue Doppler velocities were measured, and the E/A ratio was computed at both corners of the mitral annulus (16). All measurements were made by the same examiner under blind conditions.

\section{Statistical analysis}

All statistical analyses were performed using the JMP 9.0 software program (SAS Institute, Cary, NC, USA). Continuous data were expressed as means $\pm \mathrm{SD}$, and categorical data were expressed as a number (percentage). Comparisons between the two groups were made by the Student's $t$-test 
Table 1. Baseline Clinical Characteristics of the Study Population

\begin{tabular}{|c|c|c|c|}
\hline & LAVI $<32 \mathrm{~mL} / \mathrm{m}^{2} \quad(\mathrm{n}=146)$ & $\mathrm{LAVI}>32 \mathrm{~mL} / \mathrm{m}^{2}(\mathrm{n}=28)$ & $\mathrm{p}$ value \\
\hline Age (years) & $63.3 \pm 11.9$ & $63.3 \pm 13.5$ & 0.9858 \\
\hline Gender (male, $\mathrm{n})(\%)$ & $94(64.4 \%)$ & $19(67.9 \%)$ & 0.7242 \\
\hline $\operatorname{BSA}\left(\mathrm{m}^{2}\right)$ & $1.5 \pm 0.2$ & $1.6 \pm 0.2$ & 0.4093 \\
\hline Diabetes (n, \%) & $66(45.2 \%)$ & $12(42.9 \%)$ & 0.8190 \\
\hline History of cardio vascular disease $(n, \%)$ & $33(22.6 \%)$ & $12(42.9 \%)$ & 0.0250 \\
\hline Hypertension (n, \%) & $138(94.5 \%)$ & $23(82.1 \%)$ & 0.0225 \\
\hline Smoking & $28(19.2 \%)$ & $3(10.7 \%)$ & 0.2837 \\
\hline Duration of dialysis (months) & $105.4 \pm 106.2$ & $95.0 \pm 83.3$ & 0.6253 \\
\hline Systolic blood pressure $(\mathrm{mmHg})$ & $154.1 \pm 24.5$ & $157.4 \pm 29.5$ & 0.5332 \\
\hline Diastolic blood pressure (mmHg) & $80.3 \pm 13.2$ & $82.0 \pm 18.4$ & 0.5744 \\
\hline Urea nitrogen $(\mathrm{mg} / \mathrm{dL})$ & $68.7 \pm 15.5$ & $79.8 \pm 47.1$ & 0.0295 \\
\hline $\mathrm{Kt} / \mathrm{V}$ & $1.1 \pm 0.3$ & $0.9 \pm 0.2$ & 0.0172 \\
\hline C-reactive protein $(\mathrm{mg} / \mathrm{dL})$ & $0.6 \pm 1.1$ & $0.7 \pm 0.9$ & 0.5277 \\
\hline Albumin (g/dL) & $3.7 \pm 0.3$ & $3.6 \pm 0.4$ & 0.2027 \\
\hline Hemoglobin (g/dL) & $10.9 \pm 7.0$ & $10.2 \pm 1.1$ & 0.6034 \\
\hline Calcium (mg/dL) & $9.4 \pm 0.9$ & $9.4 \pm 1.1$ & 0.7716 \\
\hline Phosphate (mg/dL) & $5.4 \pm 1.5$ & $5.5 \pm 1.8$ & 0.7877 \\
\hline Intact-parathyroid hormone $(\mathrm{pg} / \mathrm{mL})$ & $200.7 \pm 195.1$ & $202.0 \pm 280.4$ & 0.9765 \\
\hline \multicolumn{4}{|l|}{ Medication (n, \%) } \\
\hline $\mathrm{ARB}$ or ACEI & $77(52.7 \%)$ & $8(28.6 \%)$ & 0.0191 \\
\hline Calcium channel blocker & $88(60.3 \%)$ & $17(60.7 \%)$ & 0.9652 \\
\hline Statin & $16(11.0 \%)$ & $1(3.6 \%)$ & 0.2278 \\
\hline Sevelamer & $16(11.0 \%)$ & $6(21.4 \%)$ & 0.1268 \\
\hline Oral active vitamin $D_{3}$ & $42(28.8 \%)$ & $8(28.6 \%)$ & 0.9833 \\
\hline
\end{tabular}

or the chi-squared test. The relationship between paired variables was analyzed by calculating the Pearson sample correlation coefficient. The independent prognostic value of LAVI for CV mortality was analyzed by multiple Cox regression analysis. The estimated regression coefficients and standard errors in the Cox regression analysis were used to calculate the hazard ratios (HR) and 95\% confidence intervals (CIs). Survival was estimated on the basis of the Kaplan-Meier curves, and the survival data were compared using the logrank test. The predictive value for all-cause mortality and $\mathrm{CV}$ mortality was analyzed by receiver operating characteristic (ROC) curve analysis and calculation of the area under the ROC curve (AUC). A two-sided $\mathrm{p}$ value $<0.05$ was used as the criterion for statistical significance.

\section{Results}

The baseline characteristics of the study population are shown in Table 1. Of the 174 patients, 28 patients (16.1\%) had an LAVI $>32 \mathrm{~mL} / \mathrm{m}^{2}$ and were considered to have an elevated LAV. Compared to patients with LAVI $\leq 32 \mathrm{~mL} /$ $\mathrm{m}^{2}$, those with The LAVI $>32 \mathrm{~mL} / \mathrm{m}^{2}$ group had a higher past history rate of CVD $(42.9 \%$ vs. $22.6 \%, \mathrm{p}=0.0250)$ and lower $\mathrm{Kt} / \mathrm{V}$ values $(0.9 \pm 0.2$ vs. $1.1 \pm 0.3, \mathrm{p}=0.0172)$ than the LAVI $\leq 32 \mathrm{~mL} / \mathrm{m}^{2}$ group. In addition, ARBs or ACEIs were prescribed for a significantly smaller proportion of the patients with LA dilatation $(28.6 \%$ vs. $52.7 \%, \mathrm{p}=0.0191)$. There were no differences between the two groups in age, gender, prevalence of diabetes, systolic or diastolic BP, smoking status, dialysis vintage, serum albumin, CRP, cal- cium, phosphate, or iPTH level, hemoglobin concentration, or use of statins, sevelamer, or active vitamin D (Table 1).

The differences in echocardiographic parameters between the two groups of patients are shown in Table 2. LVMI $\left(192.6 \pm 48.1\right.$ vs. $\left.263.0 \pm 77.3 \mathrm{~g} / \mathrm{m}^{2}, \mathrm{p}<0.001\right)$ was elevated in the LAVI $>32 \mathrm{~mL} / \mathrm{m}^{2}$ group, but there was no difference in E/A ratio between the two groups. Also, EF $(60.4 \pm 10.3$ vs. $52.9 \pm 17.1 \%, \mathrm{p}=0.0022$ ) was lower in the LAVI $>32 \mathrm{~mL} / \mathrm{m}^{2}$ group. LAVI was positively correlated with LVMI ( $\mathrm{r}=$ $0.5255, \mathrm{p}<0.0001$, Fig. $1 \mathrm{~A})$ and inversely correlated with EF $(\mathrm{r}=-0.2239, \mathrm{p}=0.0032)$ (Fig. 1B).

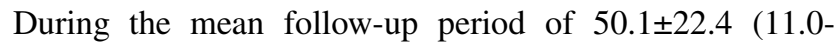
80.4) months, 77 patients $(44.3 \%)$ died, $35(45.5 \%)$ of them from $\mathrm{CV}$ causes, which consisted of cerebrovascular disease in 11 patients, sudden cardiac death in 7 patients, acute myocardial infarction in 7 patients, CHF in 7 patients, and peripheral artery disease in 3 patients. The cause of death in the other 42 patients was a malignancy in 12 patients, pneumonia in 10 patients, sepsis in 7 patients, gastrointestinal bleeding in 6 patients, ischemic colitis in 2 patients, and acute pancreatitis in 1 patient, and it was unknown in 4 patients. The all-cause mortality $(\mathrm{p}=0.0033$, Fig. $2 \mathrm{~A})$ and $\mathrm{CV}$ mortality $(p=0.0071$, Fig. $2 B$ ) were significantly lower in the LAVI $\leq 32 \mathrm{~mL} / \mathrm{m}^{2}$ group than in the LAVI $>32 \mathrm{~mL} / \mathrm{m}^{2}$ group. In addition, the all-cause mortality $(\mathrm{p}=0.0280$, Fig. 3A) was significantly lower in the EF $<55 \%$ group than in the $\mathrm{EF} \geq 55 \%$ group, and there was also significant difference in the survival rate due to $\operatorname{CVD}(p=0.0005$, Fig. 3B).

Multivariate analysis adjusted for echocardiographic pa- 
Table 2. Echocardiographic Parameters in the Study Population

\begin{tabular}{lccc}
\hline & LAVI $<32 \mathrm{~mL} / \mathrm{m}^{2}(\mathrm{n}=146)$ & LAVI $>32 \mathrm{~mL} / \mathrm{m}^{2}(\mathrm{n}=28)$ & $\mathrm{p}$ value \\
\hline EF $(\%)$ & $60.4 \pm 10.3$ & $52.9 \pm 17.1$ & 0.0022 \\
LVDd $(\mathrm{mm})$ & $48.9 \pm 7.8$ & $56.3 \pm 7.6$ & $<0.0001$ \\
LAD $(\mathrm{mm})$ & $36.6 \pm 5.7$ & $49.9 \pm 5.1$ & $<0.0001$ \\
LAD index $\left(\mathrm{mm} / \mathrm{m}^{2}\right)$ & $23.9 \pm 4.0$ & $32.0 \pm 3.5$ & $<0.0001$ \\
LVM $(\mathrm{g})$ & $300.0 \pm 83.3$ & $412.3 \pm 110.7$ & $<0.0001$ \\
LVM index $\left(\mathrm{g} / \mathrm{m}^{2}\right)$ & $192.6 \pm 48.1$ & $263.0 \pm 77.3$ & $<0.0001$ \\
LAV $(\mathrm{mL})$ & $27.5 \pm 11.7$ & $67.0 \pm 22.3$ & $<0.0001$ \\
LAV index $\left(\mathrm{mL} / \mathrm{m}^{2}\right)$ & $17.8 \pm 7.2$ & $42.1 \pm 10.8$ & $<0.0001$ \\
E/A & $0.9 \pm 0.5$ & $0.9 \pm 0.3$ & 0.8049 \\
\hline
\end{tabular}

EF: ejection fraction, LVDd: left ventricular end-diastolic diameter, LAD: left atrial diameter, LVM: Left ventricular mass, LAV: left atrial volume, E/A: mitral early (E) and late (A) inflow velocity ratio.

A

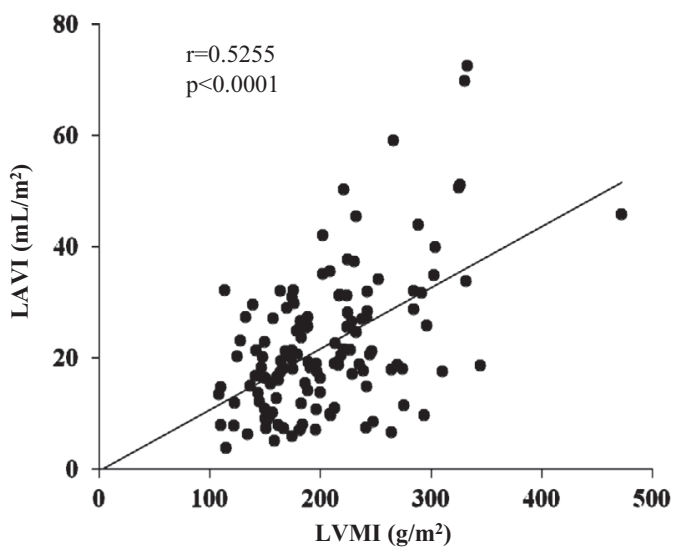

B

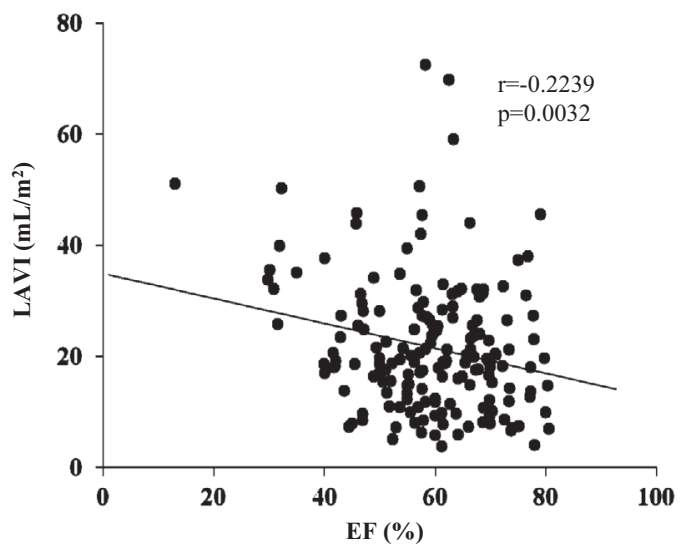

Figure 1. Relationship between LA volume index (LAVI) with left ventricular mass index (LVMI) (A) and EF. Data are correlation coefficient (r) and p values.

rameters, presence of diabetes and hypertension, history of CVD, serum urea nitrogen level and prescription of an ACEI and/or ARB showed that an increased LAVI was an independent predictor of all-cause mortality (HR 1.030, 95\% CI 1.004-1.056, $\mathrm{p}=0.0260$ ). In addition, decreased EF (HR $0.969,95 \%$ CI $0.946-0.994, \mathrm{p}=0.0153)$ and the presence of diabetes (HR 1.996, 95\% CI 1.111-3.612, $\mathrm{p}=0.0209$ ) were significantly associated with an increased risk of all-cause mortality (Table 3). However, the LAVI was not significantly association with CV mortality $(\mathrm{p}=0.1858)$, while the $\mathrm{EF}$ was significantly associated with $\mathrm{CV}$ mortality ( $\mathrm{p}=$ 0.0007) (Table 4).

As shown Fig. 4A, increased LAVI had a similar predictive value for all-cause ( $\mathrm{AUC}=0.612, \mathrm{p}=0.0059$ ) when compared with $\mathrm{EF}(\mathrm{AUC}=0.597, \mathrm{p}=0.0200)$ and LVMI (AUC= $0.655, \mathrm{p}=0.0032)$. In addition, increased LAVI had predictive value for $\mathrm{CV}$ mortality $(\mathrm{AUC}=0.581, \mathrm{p}=0.0494$ ) as shown in Fig. 4B.

\section{Discussion}

The results of the present study showed that increased LAVI was an independent risk factor for all-cause mortality in HD patients. Three of the echocardiographic parameters, increased LAVI as well as EF and LVMI, had significant predictive value for all-cause mortality but not for CV mortality.

Recently, much attention has been focused on LA volume, in addition to LVH and LV systolic dysfunction as an indicator of $\mathrm{CV}$ outcomes. LA dilation induces stasis and the development of atrial fibrillation, which predisposes the patient to thromboembolism (17). Atrial stretch facilitates neurohormonal activation and the secretion of atrial natriuretic peptide, which may have a role in the development of atrial dysrhythmias. The loss of organized atrial activity can also directly precipitate CHF (18). The LA is not only a passive conduit for blood to the LV, but it also affects LV filling because of its contractile function. The LA contributes up to $30 \%$ of the total LV stroke volume in healthy individuals, and this atrial contribution is particularly important to maintaining adequate LV stroke volume in the presence of LV dysfunction (5).

Previous studies have reported that an enlarged LA is predictive of an adverse outcome in patients with CV conditions such as cardiomyopathy (19) or acute myocardial infarction (20). However, few studies have evaluated the relationship between LAVI values and CV outcomes in ESRD patients. Ozdogan et al. evaluated the predictive value of in- 
A

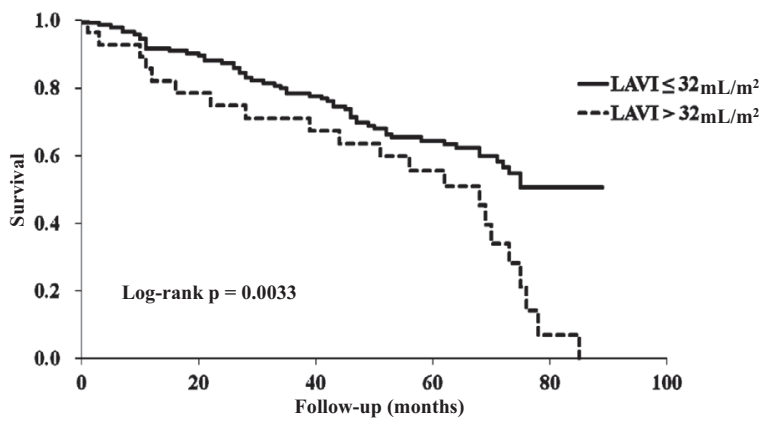

B

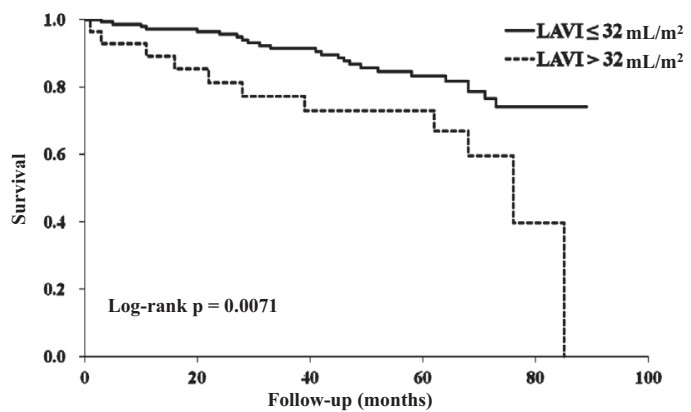

Figure 2. Kaplan-Meier survival curves for all-cause mortality (A) and cardiovascular mortality (B) according to LA volume index (LAVI).

A

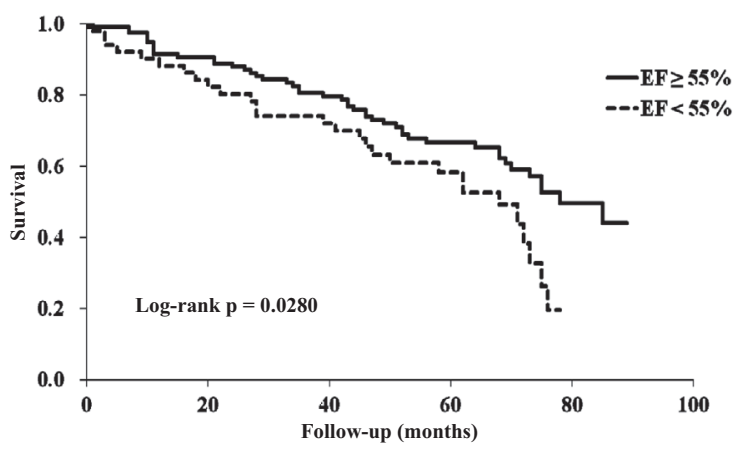

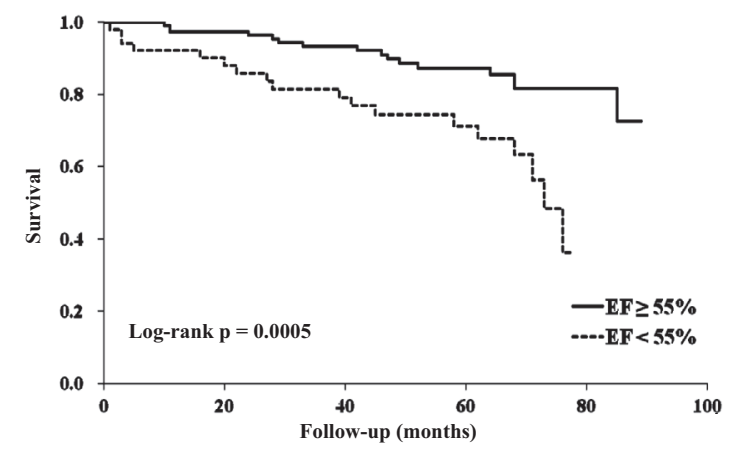

Figure 3. Kaplan-Meier survival curves for all-cause mortality (A) and cardiovascular mortality (B) according to EF.

Table 3. Multivariate Cox Regression Models of LAVI for All-cause Mortality

\begin{tabular}{lccc}
\hline & HR & $95 \%$ CI & p value \\
\hline LAVI & 1.030 & $1.004-1.056$ & 0.0260 \\
EF & 0.969 & $0.946-0.994$ & 0.0153 \\
LVMI & 0.995 & $0.988-1.002$ & 0.1844 \\
Diabetes & 1.996 & $1.111-3.612$ & 0.0209 \\
ACEI/ARB & 1.084 & $0.569-2.098$ & 0.8071 \\
Hypertension & 0.362 & $0.125-1.123$ & 0.0774 \\
Serum urea nitrogen & 1.002 & $0.986-1.014$ & 0.7904 \\
History of CVD & 1.019 & $0.513-1.937$ & 0.9561 \\
\hline
\end{tabular}

LAVI: left atrial volume index, EF: ejection fraction, LVMI: left ventricular mass index, ACEI: angiotensin-converting enzyme inhibitor, ARB: angiotensin receptor blocker,

CVD: cardiovascular disease

creased LA volume in ESRD patients, but since most of their study population were $\operatorname{HD}$ patients $(21,22)$, and LV systolic and diastolic function was not included in the multivariable Cox regression analysis, it was uncertain whether LAVI predicted an adverse outcome independent of the other echocardiographic parameters. The present study clearly showed that increased LAVI independently predicted all-cause mortality but not for CV mortality in HD patients and had a higher predictive value among echocardiographic parameters.

In previous studies, LVH was shown to be an important prognostic factor for CV events in ESRD patients (4). An increase in LV mass and cardiac fibrosis has profound consequences in ESRD patients. Sudden cardiac death, linked to abnormal electrical conduction in the distorted and fibrotic ventricle, is a prominent fatal event in ESRD patients. In the late stage, LVH and cardiac fibrosis lead to both diastolic and systolic dysfunction, and ultimately to clinically recognizable CHF, which has a definite adverse effect on the long-term survival of ESRD patients (23). Moreover, LAVI was not considered in the analyses.

On the other hand, LA size has been reported to be a 
Table 4. Multivariate Cox Regression Models of LAVI for Cardiovascular Mortality

\begin{tabular}{lccc}
\hline & HR & $95 \%$ CI & p value \\
\hline LAVI & 1.025 & $0.988-1.063$ & 0.1858 \\
EF & 0.939 & $0.905-0.973$ & 0.0007 \\
LVMI & 0.992 & $0.982-1.001$ & 0.0981 \\
Diabetes & 2.689 & $1.171-6.415$ & 0.0198 \\
ACEI/ARB & 0.828 & $0.327-2.098$ & 0.6877 \\
Hypertension & 0.610 & $0.129-3.242$ & 0.5481 \\
Serum urea nitrogen & 0.999 & $0.978-1.013$ & 0.8966 \\
History of CVD & 1.659 & $0.587-4.374$ & 0.3276 \\
\hline
\end{tabular}

LAVI: left atrial volume index, EF: ejection fraction, LVMI: left ventricular mass index, ACEI: angiotensin-converting enzyme inhibitor, ARB: angiotensin receptor blocker, CVD: cardiovascular disease

A

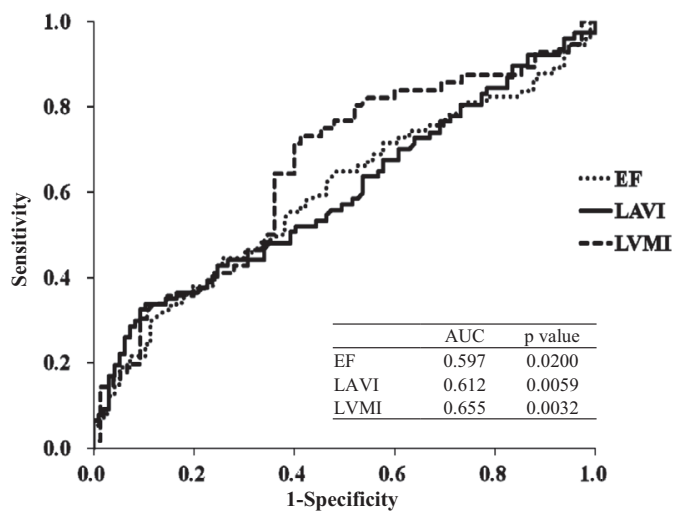

B

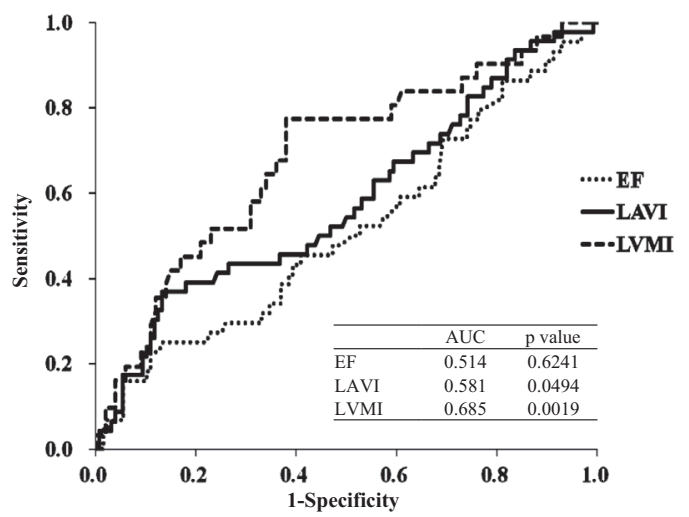

Figure 4. ROC curve analyses and AUCs calculated for all-cause mortality (A) and cardiovascular mortality (B).

more stable indicator of the duration and severity of diastolic dysfunction than any other echocardiographic parameters, even though it is largely determined by the same factors that determine diastolic LV filling (24). In addition, Moller et al. (20) showed that LAVI is superior to conventional Doppler indices of diastolic function for predicting mortality in patients with acute myocardial infarction. In line with these findings, we demonstrated that increased LAVI was an independent risk factor for all-cause mortality, whereas an elevated E/A ratio was not, suggesting that LAVI is a more reliable predictor for all-cause mortality in HD patients.

The present study had several limitations. First, there was an inherent limitation in the observational study design. Second, we could not assess other indicators of extracellular volume, such as natriuretic peptide measured by immunoassay or the extracellular fluid volume/total body water ratio measured by multifrequency electric bioimpedance. However, this assessment tool has not yet been validated in HD patients. Third, the echocardiographic parameters may have been confounding factors in relation to other parameters, because most of the values were correlated with each other. Nevertheless, LAVI had predictive value for all-cause mortality that was similar to that of EF and LVMI in a multi- variable Cox model, even after adjustment of such parameters and ROC curve analyses. Finally, the echocardiographic examination was performed only at the start of the study, and whether the echocardiographic findings remained unchanged during the follow-up period is unknown. Serial follow-up echocardiography examinations may be of help in understanding the natural history of LA remodeling in HD patients (25). Determining whether regression of LAVI is correlated with improved all-cause mortality will require further study.

In conclusion, the results of this study showed that increased LAVI predicts all-cause mortality of chronic HD patients. Our findings suggest that measurement of LAVI may be helpful in the risk stratification of HD patients and in providing therapeutic direction for their management.

\section{The authors state that they have no Conflict of Interest (COI).}

\section{Acknowledgement}

This work was in part supported by a grant of the Japan Promotion Society of Cardiovascular disease.

\section{References}

1. Parfrey PS, Foley RN. The clinical epidemiology of cardiac dis- 
ease in chronic renal failure. J Am Soc Nephrol 10: 1606-1615, 1999.

2. Cheung AK, Sarnak MJ, Yan G, et al. Atherosclerotic cardiovascular disease risks in chronic hemodialysis patients. Kidney Int $\mathbf{5 8}$ : 353-362, 2000.

3. K/DOQI clinical practice guidelines for cardiovascular disease in dialysis patients. Am J Kidney Dis 45: S1-S153, 2005.

4. London GM, Pannier B, Guerin AP, et al. Alterations of left ventricular hypertrophy in and survival of patients receiving hemodialysis: follow-up of an interventional study. J Am Soc Nephrol 12: 2759-2767, 2001

5. Leung DY, Boyd A, Ng AA, Chi C, Thomas L. Echocardiographic evaluation of left atrial size and function: current understanding, pathophysiologic correlates, and prognostic implications. Am Heart J 156: 1056-1064, 2008

6. Abhayaratna WP, Seward JB, Appleton CP, et al. Left atrial size: physiologic determinants and clinical applications. J Am Coll Cardiol 47: 2357-2363, 2006.

7. Alberti KG, Zimmet PZ. Definition, diagnosis and classification of diabetes mellitus and its complications. Part 1: diagnosis and classification of diabetes mellitus provisional report of a WHO consultation. Diabet Med 15: 539-553, 1998.

8. Daugirdas JT. Second generation logarithmic estimates of singlepool variable volume $\mathrm{Kt} / \mathrm{V}$ : an analysis of error. J Am Soc Nephrol 4: 1205-1213, 1993.

9. Schiller NB, Shah PM, Crawford M, et al. Recommendations for quantitation of the left ventricle by two-dimensional echocardiography. American Society of Echocardiography Committee on Standards, Subcommittee on Quantitation of Two-Dimensional Echocardiograms. J Am Soc Echocardiogr 2: 358-367, 1989.

10. Otterstad JE, Froeland G, St John, Sutton M. Accuracy and reproducibility of biplane two-dimensional echocardiographic measurements of left ventricular dimensions and function. Eur Heart J 18: 507-513, 1997.

11. Devereux RB, Alonso DR, Lutas EM, et al. Echocardiographic assessment of left ventricular hypertrophy: comparison to necropsy findings. Am J Cardiol 57: 450-458, 1986.

12. Liao Y, Cooper RS, Durazo-Arvizu R, Mensah GA, Ghali JK. Prediction of mortality risk by different methods of indexation for left ventricular mass. J Am Coll Cardiol 29: 641-647, 1997.

13. Sahan DJ, DeMaria A, Kisslo J, Weyman A. Recommendations regarding quantitation in M-mode echocardiography: results of a survey of echocardiographic measurements. Circulation 58: 10721083, 1978.

14. Lester SJ, Ryan EW, Schiller NB, Foster E. Best method in clinical practice and in research studies to determine left atrial size. Am J Cardiol 84: 829-832, 1999.

15. Pritchett AM, Mahoney DW, Jacobsen SJ, Rodeheffer RJ, Redfield MM. Diastolic dysfunction and left atrial volume: a populationbased study. J Am Coll Cardiol 45: 87-92, 2005.

16. Oki T, Tabata T, Yamada H, et al. Clinical application of pulsed Doppler tissue imaging for assessing abnormal left ventricular relaxation. Am J Cardiol 79: 921-928, 1997.

17. Tsang TS, Abhayaratna WP, Barnes ME, et al. Prediction of cardiovascular outcomes with left atrial size: is volume superior to area or diameter? J Am Coll Cardiol 47: 1018-1023, 2006.

18. Kizer JR, Bella JN, Palmieri V, et al. Left atrial diameter as an independent predictor of first clinical cardiovascular events in middle-aged and elderly adults: the Strong Heart Study (SHS). Am Heart J 151: 412-418, 2006.

19. Sabharwal N, Cemin R, Rajan K, Hickman M, Lahiri A, Senior R. Usefulness of left atrial volume as a predictor of mortality in patients with ischemic cardiomyopathy. Am J Cardiol 94: 760-763, 2004.

20. Moller JE, Hillis GS, Oh JK, et al. Left atrial volume: a powerful predictor of survival after acute myocardial infarction. Circulation 107: 2207-2212, 2003.

21. Ozdogan O, Kayikcioglu M, Asci G, et al. Left atrial volume predicts mortality in low-risk dialysis population on long-term lowsalt diet. Am Heart J 159: 1089-1094, 2010.

22. Tripepi G, Benedetto FA, Mallamaci F, Tripepi R, Malatino L, Zoccali C. Left atrial volume in end-stage renal disease: a prospective cohort study. J Hypertens 24: 1173-1180, 2006.

23. Glassock RJ, Pecoits-Filho R, Barberato SH. Left ventricular mass in chronic kidney disease and ESRD. Clin J Am Soc Nephrol 4 (Suppl 1): S79-S91, 2009.

24. Simek CL, Feldman MD, Haber HL, Wu CC, Jayaweera AR, Kaul S. Relationship between left ventricular wall thickness and left atrial size: comparison with other measures of diastolic function. $\mathbf{J}$ Am Soc Echocardiogr 8: 37-47, 1995.

25. Tripepi G, Mattace-Raso F, Mallamaci F, et al. Biomarkers of left atrial volume: a longitudinal study in patients with end stage renal disease. Hypertension 54: 818-824, 2009.

(C) 2012 The Japanese Society of Internal Medicine http://www.naika.or.jp/imindex.html 